\title{
Robust Input Shaper Design using Linear Matrix Inequalities
}

\author{
Thomas Conord and Tarunraj Singh \\ tconordebuffalo.edu tsingh@buffalo.edu \\ State University of New York at Buffalo, Buffalo, NY 14260
}

\begin{abstract}
This paper proposes an Linear Matrix Inequality based problem formulation to determine input shaped profiles. The cost function is the residual energy, a quadratic function of the amplitude of the shaped profile, for each sampling interval. The Schur complement permits representing the quadratic function as a Linear Matrix Inequality. Augmenting the state space model with the sensitivity of the states to uncertain parameters, input shaped profiles which are robust to model uncertainties can be derived. Finally, a minimax input shaped profile which minimizes the maximum magnitude of the residual energy over the domain of uncertainties is determined using the LMI problem. The proposed technique is illustrated on the single spring-mass-dashpot example. The solutions derived are shown to coincide with the solutions presented in the literature, without the requirement of solving a nonlinear programming problem.
\end{abstract}

\section{INTRODUCTION}

Shaping reference input to mitigate the effect of underdamped system response has been of interest over the past two decades. The Input-Shaping/Time-Delay controller [1] provided a simple technique to minimize the magnitude of residual vibration in the presence of model uncertainties. The robustness is achieved by minimizing the sensitivity of the residual energy to the nominal uncertain parameters. The extra-insensitive input shaper [3], and the minimax approach [2] to design controllers which incorporate knowledge of the domain of uncertainty have been proposed. These approaches are formulated as optimization problems which are computationally expensive and sensitive to initial guess since the cost function include multiple local minima. It can be surmised that with the increase in the number of uncertain parameters, the computational cost of determining the minimax solution will be significant. This provides a motivation to formulate a convex optimization problem which guarantees the determination of the globally optimal solution.

Solving optimization problem with Linear Matrix Inequalities (LMI) constraints [4] has recently resulted in algorithms which can efficiently solve large scale problems. Tools such as Sostools [5] which can synthesize Lyapunov functions for nonlinear polynomial systems by exploiting semi-definite programming, have been developed. A semi-definite program is of the form:

$$
\begin{array}{cc}
\text { minimize } & c^{T} x \\
\text { subject to } & F_{0}+\sum_{i=1}^{n} x_{i} F_{i} \succeq 0
\end{array}
$$

where $x=\left[\begin{array}{llll}x_{1} & x_{2} & \ldots & x_{n}\end{array}\right] \in R^{n}$ is a vector of variables to be optimized for and $F_{i}$ are symmetric matrices. The cost function is a linear combination of the variable $x$. The symbol $\succeq$ represents the positive semi-definite constraint. A variety of control problems can be posed in the LMI framework [4]. This paper deals with the design of input shaped control profile by exploiting the strengths of semidefinite programming. In section II, the problem of design of a input shaped profile to force the magnitude of residual energy to lie below a pre-specified threshold is presented. The proposed technique is illustrated on a single spring-mass system in section III. The is followed by the description of two techniques to design control profiles which are insensitive to uncertainties in model parameters. The paper concludes with some remarks in the final section.

\section{Problem Formulation}

The goal of designing shaped input control profile to minimize residual energy of maneuvering systems is considered in this section. Consider a linear system represented by the equations:

$$
\begin{gathered}
\dot{x}(t)=A x(t)+B u(t) \\
y(t)=C x(t)+D u(t) .
\end{gathered}
$$

Parameterizing the control profile as the output of a timedelay filter subject to a unit step input, one can represent the control input as:

$$
u(s)=\frac{1}{s}\left(A_{0}+A_{1} e^{-s T_{1}}+A_{2} e^{-s T_{2}}+\ldots+A_{N} e^{-s T_{N}}\right)
$$

Generally both the amplitudes $A_{i}$ and the time delays $T_{i}$ constitute the design variables. To permit posing the design problem in a LMI framework, the variables corresponding to the delay times are assumed known and are selected to be integer multiples of a specified delay time $T_{s}$.

The optimization problem can now be stated as the determination of the variables $A_{0}, A_{1}, \ldots, A_{N}$ to minimize the residual energy of the system at the final time $T_{N}=N T_{s}$ :

$$
\min _{A_{0}, A_{1}, \ldots, A_{N}} J=\left(x\left(T_{N}\right)-x_{f}\right)^{T} Q\left(x\left(T_{N}\right)-x_{f}\right)
$$

where $x_{f}$ is the desired final state, $x\left(T_{N}\right)$ is the actual final state of the system at the desired final time $T_{N}=N \times T_{s}$, and $Q$ is a positive definite weighting matrix. In this work, its form is one which results in $J$ representing the sum of the kinetic and potential energies in the system.

Define $\Delta x=x\left(T_{N}\right)-x_{f}$ which represents the excursion of the final states from the desired states, to permit a terse representation of the residual energy. Since the cost function 
$J$ is a quadratic function of the parameters to be optimized for, the problem is rewritten as the minimization of $\gamma$ which appears linearly in the cost function subject to the constraint that the residual energy is bounded by $\gamma$ :

$$
\min J=\gamma
$$

subject to

$$
\begin{aligned}
& \gamma-\Delta x^{T} Q \Delta x>0 \\
& A_{l b} \leq A_{i} \leq A_{u b}
\end{aligned}
$$

where $A_{l b}$ and $A_{u b}$ correspond to the lower and upper bounds of the variable $A_{i}$ respectively. The Schur complement [4] permits rewriting the optimization problem as

$$
\min J=\gamma
$$

subject to

$$
\begin{aligned}
& \left(\begin{array}{cc}
\gamma & \Delta x^{T} \\
\Delta x & Q^{-1}
\end{array}\right) \succ 0 \\
& A_{l b} \leq A_{i} \leq A_{u b}
\end{aligned}
$$

This is an LMI as as long as the variables $A_{i}$ are affine in $\Delta x$.

The error states at the final time can be represented as:

$$
\begin{aligned}
\Delta x= & x\left(T_{N}\right)-x_{f} \\
& =e^{A T_{N}} x(0)+\int_{0}^{T_{N}} e^{A\left(T_{N}-t\right)} B u(t) d t-x_{f}
\end{aligned}
$$

Let $x(0)=0$, and $u(\tau)=\tilde{A}_{i}=A_{0}+\ldots+A_{i}$ where $\tau \in$ $\left[i \times T_{s} ;(i+1) \times T_{s}\right]$ for all $i$ between 0 and $N$. By introducing these new variables $\tilde{A}_{i}$, which correspond to the effective value of the input on each interval, the terminal constraint on the control input can be represented as $\tilde{A}_{N}=1$.

With these notations, the integral in Equation 8 can be rewritten as:

$$
\Delta x=\sum_{k=0}^{N-1}\left(\int_{k \times T_{s}}^{(k+1) \times T_{s}} e^{A\left(T_{N}-\tau\right)} B \tilde{A}_{k} d \tau\right)-x_{f}
$$

This is equivalent to, by changing the variable $\tau$ into $\tau^{\prime}=$ $\tau-k \times T_{s}$ (and reminding that $T_{i}=i \times T_{s}$ for all $i$ between 0 and $N)$ :

$$
\Delta x=\sum_{k=0}^{N-1}\left(\int_{0}^{T_{s}} e^{A\left((N-k) \times T_{s}-\tau^{\prime}\right)} B d \tau^{\prime}\right) \tilde{A}_{k}-x_{f}
$$

or, noticing that $\tilde{A}_{k}$ is a scalar:

$$
\begin{aligned}
\Delta x= & \left(\sum_{k=0}^{N-1} \tilde{A}_{k} e^{A(N-k-1) \times T_{s}}\right)\left(\int_{0}^{T_{s}} e^{A\left(T_{s}-\tau\right)} B d \tau\right) \\
& -x_{f}
\end{aligned}
$$

It is clear that, from the equation 9, the magnitudes of the input appear affinely in the expression of $\Delta x$. Therefore the problem $7 \mathrm{~b}$ is an LMI for the parameters $\tilde{A}_{k}$.
The sum : $S=\sum_{k=0}^{N-1} \tilde{A}_{k} e^{A(N-k-1) \times T_{s}}$ can be rewritten as a product of three matrices:

$$
\begin{aligned}
S & =\mathcal{I U H} \\
\mathcal{I} & =\left[\begin{array}{ccc}
I & \ldots & I
\end{array}\right] \\
U= & {\left[\begin{array}{ccc}
\tilde{A}_{0} I & 0 & 0 \\
0 & \ddots & 0 \\
0 & 0 & \tilde{A}_{N-1} I
\end{array}\right] } \\
H= & {\left[\begin{array}{c}
\left(e^{A T_{s}}\right)^{N-1} \\
\vdots \\
e^{A T_{s}} \\
I
\end{array}\right] }
\end{aligned}
$$

where the three matrices $\mathcal{I}, U$, and $H$ are three matrices of dimensions $n \times(n N),(n N) \times(n N)$ and $(n N) \times n$.

Finally, the expression for $\Delta x$ requires the integral $\int_{0}^{T_{s}} e^{A\left(T_{s}-\tau\right)} B d \tau$ to be solved for in the equation 11 . This has to be computed for any matrix $A$, non-singular or not since this approach has to work now for any system stable or marginally stable. The Van Loan Identity permit calculation of the convolution integral by constructing the matrix:

$$
P=\left(\begin{array}{cc}
A & B \\
0 & 0
\end{array}\right)
$$

whose matrix exponential,

$$
e^{P T_{s}}=\left(\begin{array}{c}
e^{A T_{s}} \underbrace{\int_{0}^{T_{s}} e^{A\left(T_{s}-\tau\right)} B d \tau}_{E} \\
0
\end{array}\right)
$$

results in the convolution integral. Let $E$ be equal to $\int_{0}^{T_{s}} e^{A\left(T_{s}-\tau\right)} B d \tau$ as defined above.

The final expression of $\Delta x$ is:

$$
\Delta x=\mathcal{I} U H E-x_{f}
$$

where $U$ is the unknown matrix, and all the other matrices are given or computed easily from the state-space model of the system. The problem $7 \mathrm{~b}$ set up with this expression of $\Delta x$ is finally a very neat LMI problem which can easily be solved with the MATLAB LMI toolbox. This approach is used to design delay filters for any kind of system. Imposing the control of the bounds of the input is straight forward since the unknown corresponds directly to the value of the input on each interval.

\section{EXAMPLE}

To test this new method of designing input shaped profiles, it is applied to the usual benchmark problem which is the following single spring-mass-dashpot system:

The equations of motion:

$$
m_{1} \ddot{x}_{1}=-k x_{1}-c \dot{x}_{1}+k u
$$

can be represented in state-space form:

$$
\dot{x}=A x+B u
$$




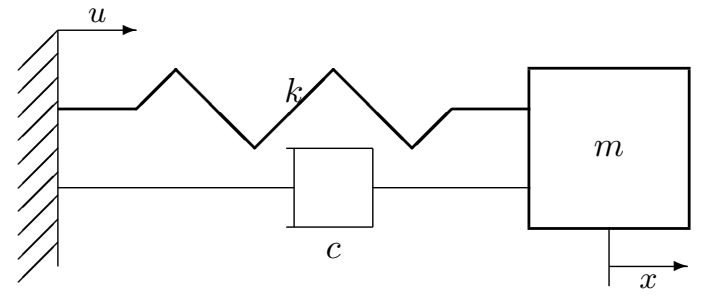

Fig. 1. Spring-Mass-Dashpot

where

$A=\left(\begin{array}{cc}0 & 1 \\ -\frac{k}{m_{1}} & -\frac{c}{m_{1}}\end{array}\right), \quad B=\left(\begin{array}{c}0 \\ \frac{k}{m_{1}}\end{array}\right), \quad x=\left(\begin{array}{c}x_{1} \\ \dot{x}_{1}\end{array}\right)$

The value of the different parameters are: $k=1, m_{1}=1$, and $c=0$. The stiffness is uncertain and lies in the range 0.7 to 1.3 . The LMI process described earlier requires the maneuver time and the number of intervals the maneuver time is discretized into, to be specified. An arbitrary selection of the maneuver time does not guarantee that the system response will be characterized by zero residual energy nor does it minimize the time to complete the maneuver. Starting with an estimate of the region within which the optimal maneuver time is resident, the bisection algorithm can be used to iteratively halve the interval to determine the optimal maneuver time. Figure 2 illustrates the variation in the magnitude of the residual energy as a function of the maneuver time. It is clear that the bisection algorithm, or the golden section can be used to determine the smallest time which satisfies the requirement that the residual energy lie below a specified threshold.

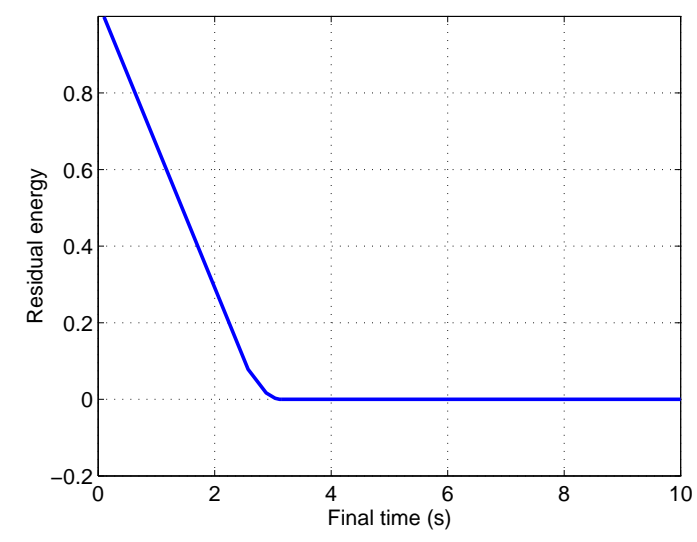

Fig. 2. Residual Energy Variation

The problem of design of the reference profile with the constraint $0<A_{i}<1$, which corresponds to $\tilde{A}_{i}<\tilde{A}_{i+1}$ results in the control profile which is identical to the $\mathrm{ZV}$ input shaper or the single time-delay filter [1]. The dashed line in Figure 3 illustrates the staircase reference profile which satisfies the boundary conditions for a maneuver time of 10 sec. The solid lines illustrate the optimal solution resulting from the bisection algorithm. The single step reference profile includes an initial step of 0.5 which changes to 1.0 at a time of 3.14 which corresponds to half the period of oscillation of the undamped system.

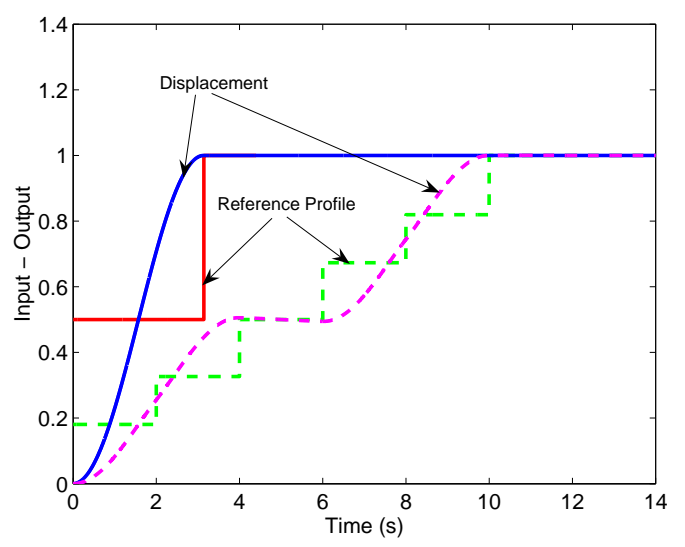

Fig. 3. Displacement/Control Profiles

The constraint $0<\tilde{A}_{i}<1$ requires a simple reformulation of the problem where the variables to be optimized for correspond to the amplitude of the reference input at each sampling interval. Figure 4 illustrates the displacement and control profile. The maneuver time is $2.095 \mathrm{sec}$ is smaller than the solution where the reference profile increases monotonically. The optimal control profile is of a bang-bang form with the lower and upper bound of 0 and 1 respectively. The dashed line and the solid lines correspond to the solutions of the maneuver time of $10 \mathrm{sec}$ and the optimal maneuver time respectively.

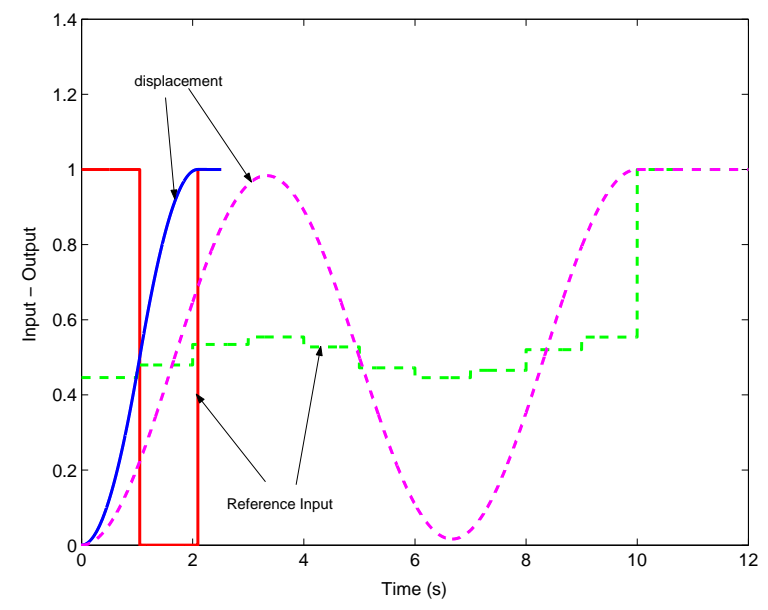

Fig. 4. Displacement/Control Profiles

\section{Robust INPUT SHAPERS}

To desensitize the reference profile to uncertainties in the spring stiffness, the state space model is augmented with the sensitivity of the states with respect to the spring stiffness. Robustness to the uncertain stiffness is achieved by forcing 
the final value of the sensitivity states to zero. The augmented model is:

$$
\dot{x}=\left[\begin{array}{cccc}
0 & 1 & 0 & 0 \\
-\frac{k}{m} & 0 & 0 & 0 \\
0 & 0 & 0 & 1 \\
-\frac{1}{m} & 0 & -\frac{k}{m} & 0
\end{array}\right] x+\left[\begin{array}{c}
0 \\
\frac{k}{m} \\
0 \\
0
\end{array}\right] u
$$

The boundary conditions for the optimization problem are:

$$
x(0)=\left\{\begin{array}{llll}
0 & 0 & 0 & 0
\end{array}\right\}, x\left(t_{f}\right)=\left\{\begin{array}{llll}
1 & 0 & 0 & 0
\end{array}\right\}
$$

Solving the LMI problem using the process described earlier results in the optimal reference profile and the corresponding displacement shown in Figure 5

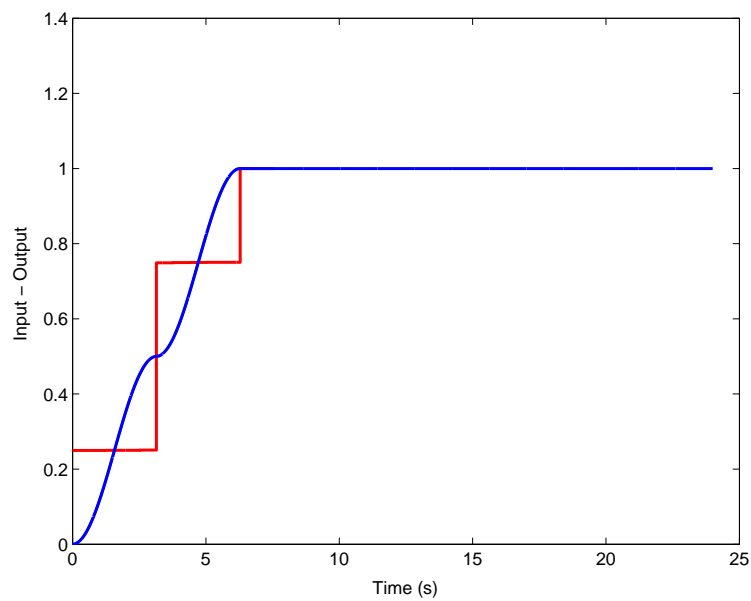

Fig. 5. Displacement/Control Profiles

To design robust controller which incorporate knowledge of the range of uncertainties, a minimax problem is formulated where the maximum magnitude of the residual energy is minimized. The problem statement is:

$$
\min J=\gamma
$$

subject to

$$
\begin{aligned}
& \left(\begin{array}{cc}
\gamma & \Delta x_{i}^{T} \\
\Delta x_{i} & Q_{i}^{-1}
\end{array}\right) \succ 0 \text { for } i=1,2, \ldots, K \\
& A_{l b} \leq A_{i} \leq A_{u b}
\end{aligned}
$$

where

$$
\begin{aligned}
\Delta x_{i}= & e^{A_{i} T_{N}} x(0)+ \\
& \sum_{j=0}^{N} \int_{T_{j}}^{T_{N}} e^{A_{i}\left(T_{N}-t\right)} B A_{i} \mathcal{H}\left(t-T_{j}\right) d t-x(20) \\
Q_{i}= & {\left[\begin{array}{rr}
k_{i} & 0 \\
0 & m
\end{array}\right] \text { for } i=1,2, \ldots, K }
\end{aligned}
$$

where $k_{i}$ represents the $i^{\text {th }}$ manifestation of the uncertain spring stiffness. This formulation increases the LMIs by a factor of $K$, the number of discretizations of the uncertain space. Figure 6 illustrates the response of the nominal plane to the reference profile generated by the minimax LMI problem.

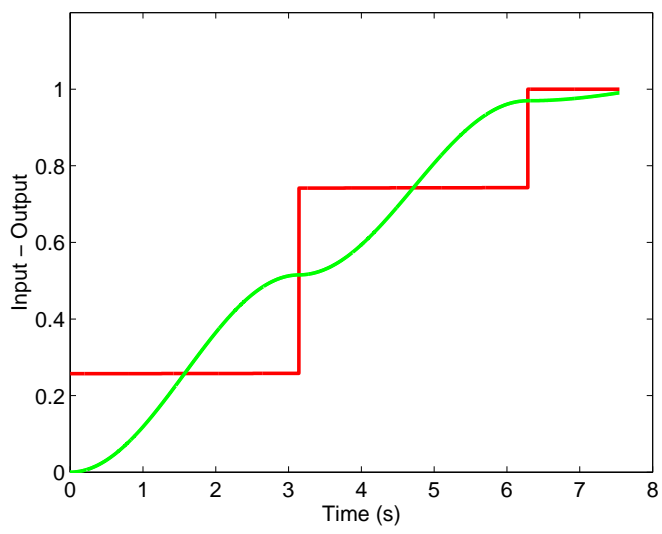

Fig. 6. Displacement/Control Profiles

The bisection approach is not used to optimize for the maneuver time. For a fair comparison with the robust controller, the same maneuver time as the robust controller is selected for the minimax controller.

Figure 7 illustrates the variation of residual energy as a function of the uncertain spring stiffness. It is clear that over the domain of uncertainty, the minimax controller has a reasonable performance compared to the robust controller

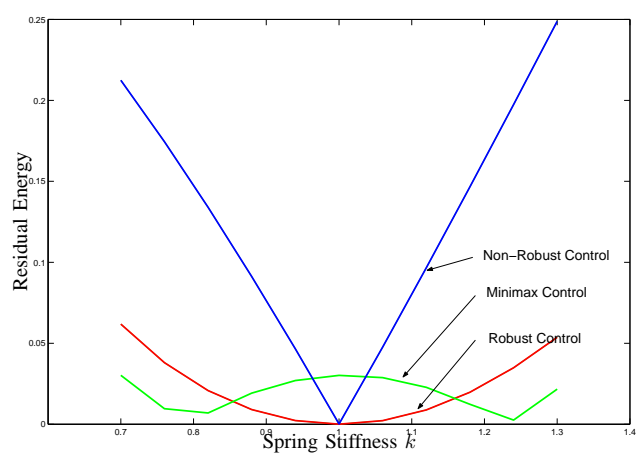

Fig. 7. Residual Energy Sensitivity

To illustrate that the proposed technique can be easily extended to higher order systems, the input shaped profiles for a single, two and three spring-mass systems were designed and the optimal control profiles and the corresponding displacements of the masses are illustrated in Figure 8. All the control profiles were parameterized by 21 control steps and the optimal maneuver times are $3.147 \mathrm{~s}, 5.666 \mathrm{~s}, 8.711 \mathrm{~s}$ respectively. The computational time for the determination of the control profiles are $2.033 \mathrm{~s}, 10.407 \mathrm{~s}, 30.585 \mathrm{~s}$ respectively which is not a linear function of the number of states, but is reasonable. 


\section{CONClusion}

The LMI approach is a very powerful tool which permits solving the problem of design of input shapers for systems with modelling uncertainties. It provides a convenient mechanism to convert a quadratic cost function into a LMI. The benefit of converting the minimax optimization problem which is a nonlinear programming problem into a semi-definite programming problem is the preclusion of the necessity of a good initial guess to determine the globally optimal solution.

This paper illustrates the use of the LMI problem formulation in conjunction with a bisection algorithm to determine a input shaped control profile which minimizes the time necessary to eliminate residual vibrations of the systems. Techniques to desensitize the control profile to modelling uncertainties is illustrated by augmenting the system with the sensitivity states and by posing a minimax problem. The proposed technique is illustrated on a single spring-mass system where the spring stiffness is assumed to be uncertain but with known bounds. The resulting control profiles are identical to the ones presented in the literature.

\section{REFERENCES}

[1] Tarunraj Singh and Willaim Singhose, Tutorial on Input Shaping/Time Delay Control of Maneuvering Flexible Structures, Proceedings of the 2002 American Control Conference, Anchorage, Alaska.

[2] T. Singh, Minimax Design of Robust Controllers for Flexible Systems, Journal of Guidance, Control and Dynamics, vol. 25, No. 5, pp. 868875, 2002.

[3] Singhose, W., Derezinski, S. and Singer, N., "Extra-Insensitive Input Shapers for Controlling Flexible Spacecraft", AIAA J. of Guidance, Control, and Dynamics, vol. 19, pp. 385-91, 1996.

[4] Boyd S., El Ghaoui, L., Feron, E., and Balakrishnan, V., "Linear Matrix Inequalities in System \& Control Theory (Studies in Applied Mathematics, Volume 15)", Society for Industrial and Applied Mathematics (SIAM), July 1994.

[5] Prajna, S., Papachristodoulou, A., Seiler, P. and Parrilo, P. A., SOSTOOLS: Sum of squares optimization toolbox for MATLAB, http://www.cds.caltech.edu/sostools, 2004. 

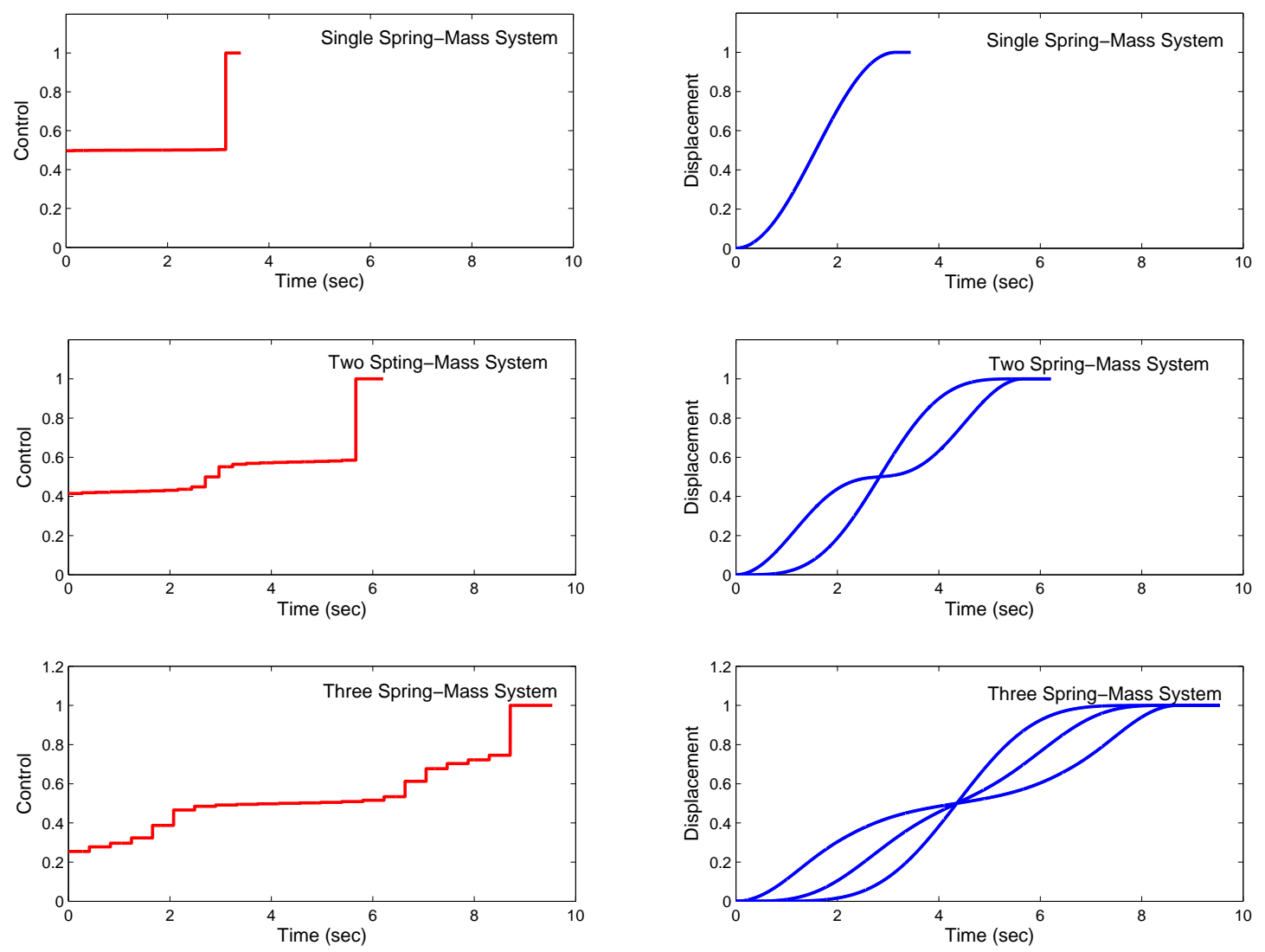

Fig. 8. Input Shaped Control and Displacement Profiles 